\title{
REVIEW
}

\section{Recent Improvements in Geant4 Electromagnetic Physics Models and Interfaces}

Vladimir IVANCHENKO ${ }^{1,2,3^{*}}$, John APOSTOLAKIS ${ }^{1}$, Alexander BAGULYA ${ }^{4}$, Haifa Ben ABDELOUAHED ${ }^{5}$, Rachel BLACK ${ }^{6}$, Alexey BOGDANOV ${ }^{7}$, Helmut BURKHARD ${ }^{1}$, Stéphane CHAUVIE ${ }^{8}$, Pablo CIRRONE ${ }^{9}$, Giacomo CUTTONE $^{9}$, Gerardo DEPAOLA ${ }^{10}$, Francesco Di ROSA ${ }^{9}$, Sabine ELLES ${ }^{11}$, Ziad FRANCIS ${ }^{12}$, Vladimir GRICHINE ${ }^{4}$, Peter GUMPLINGER ${ }^{13}$, Paul GUEYE ${ }^{6}$, Sebastien INCERTI ${ }^{14}$, Anton IVANCHENKO ${ }^{14}$, Jean JACQUEMIER ${ }^{11}$, Anton LECHNER ${ }^{1,15}$, Francesco LONGO ${ }^{16}$, Omrane KADRI ${ }^{5}$, Nicolas KARAKATSANIS ${ }^{17}$, Mathieu KARAMITROS ${ }^{14}$, Rostislav KOKOULIN ${ }^{7}$, Hisaya KURASHIGE ${ }^{18}$, Michel MAIRE ${ }^{11,19}$, Alfonso MANTERO ${ }^{20}$, Barbara MASCIALINO ${ }^{21}$, Jakub MOSCICKI ${ }^{1}$, Luciano PANDOLA ${ }^{22}$, Joseph PERL ${ }^{23}$, Ivan PETROVIC ${ }^{9}$, Aleksandra RISTIC-FIRA ${ }^{9}$, Francesco ROMANO ${ }^{9}$, Giorgio RUSSO ${ }^{9}$, Giovanni SANTIN ${ }^{24}$, Andreas SCHAELICKE ${ }^{25}$, Toshiyuki TOSHITO ${ }^{26}$, Hoang TRAN ${ }^{14}$, Laszlo URBAN ${ }^{19}$, Tomohiro YAMASHITA ${ }^{27}$ and Christina ZACHARATOU ${ }^{28}$

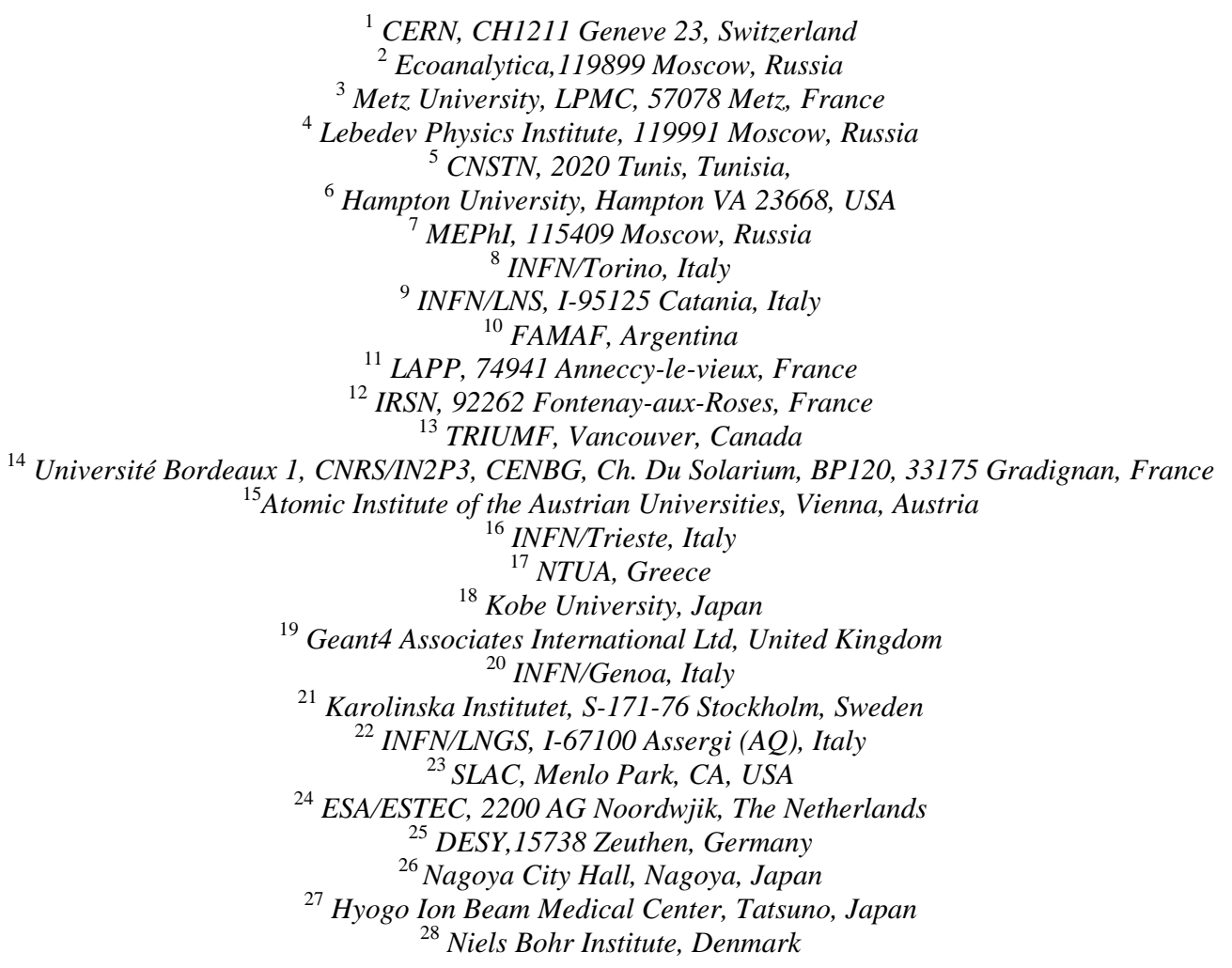

\begin{abstract}
An overview of the electromagnetic (EM) physics of the Geant4 toolkit is presented. Two sets of EM models are available: the "Standard" initially focused on high energy physics (HEP) while the "Low-energy" was developed for medical, space and other applications. The "Standard" models provide a faster computation but are less accurate for $\mathrm{keV}$ energies, the "Low-energy" models are more CPU time consuming. A common interface to EM physics models has been developed allowing a natural combination of ultra-relativistic, relativistic and low-energy models for the same run providing both precision and CPU performance. Due to this migration additional capabilities become available. The new developments include relativistic models for bremsstrahlung and e+e- pair production, models of multiple and single scattering, hadron/ion ionization, microdosimetry for very low energies and also improvements in existing Geant4 models. In parallel, validation suites and benchmarks have been intensively developed.
\end{abstract}

KEYWORDS: Geant4, bremsstrahlung, Monte Carlo, multiple scattering, energy loss

\section{Introduction}

Geant4 is a general purpose toolkit ${ }^{1,2)}$ for Monte Carlo si-

*Corresponding author, E-mail:Vladimir.Ivantchenko@cern.ch

(C) 2011 Atomic Energy Society of Japan, All Rights Reserved. mulation of particle transport in matter. The standard EM package of Geant $4^{1,3-12)}$ provides simulation of EM interactions of particles in the energy interval from $1 \mathrm{keV}$ to $10 \mathrm{PeV}$. It is used for Monte Carlo production for the Large Hadron 
Collider (LHC) experiments at CERN. The precision and CPU performance of the package directly affect LHC simulation results. This package also has a lot of applications in smaller size HEP experiments and in other domains like medical and space science.

The Low-energy EM package ${ }^{2,13-18)}$ includes an alternative set of models extending Geant4 applicability at lower energy (by default down to $100 \mathrm{eV}$ ):

- models based on Livermore databases EPDL ${ }^{19)}$ and EEDL; ${ }^{20)}$

- $\quad$ Penelope ${ }^{21)}$ models implemented in $\mathrm{C}++$;

- $\quad$ the atomic de-excitation module based on Livermore database EADL. ${ }^{22)}$

This package is focused on medical and space applications and was originally developed independently of the Standard EM package. Unfortunately, this approach had some software and practical limitations which did not permit the full exploration of Geant4 capabilities. To overcome these limitations a migration of the Low-energy package to the model design ${ }^{9)}$ developed for the Standard EM package has been performed. This migration provides a boost for new model developments and validation.

In this work we describe the current status of Geant4 EM packages for the Geant4 release 9.3, selected recent developments and validation results.

\section{New Geant4 EM Interfaces}

\section{Model Design}

Geant4 physics is implemented via the general G4VProcess interface ${ }^{1)}$ for all possible interactions: transportation, decay, EM, hadronic, optical, and others. For the EM physics processes three interfaces are implemented, ${ }^{9)}$ which inherit from G4VProcess:

- G4VEnergyLossProcess,

- G4VEmProcess,

- G4VMultipleScattering.

Each of these classes performs generic computation common to all concrete processes. For example, the first interface is used for implementation of all ionization, bremsstrahlung, and $\mathrm{e}^{+} \mathrm{e}^{-}$pair production processes. Concrete physics models inherit from the G4VEmModel interface. Any process may have several models specialized for the primary particle energy and geometry region ${ }^{2)}$ (G4Region object). Thus, the migration to model interface allows the combination of models from the two EM packages for the same process. Thanks to this design, an implementation of a model requires only computation of cross sections, stopping power and sampling of a final state. All management functions are performed by these virtual classes.

The consolidation of all EM sub-packages gives a boost for the development of new models, automatically resolves a number of problems ${ }^{23}$ seen in previous versions of the Low-energy package, provides new opportunities for complex simulation of high energy and low-energy effects, and allows a deeper validation of EM physics. For example, simulated particle ranges are now stable ${ }^{24)}$ independently both of cut values and simulation conditions.

\section{EM Physics Lists}

Geant4 physics is encoded into Physics Lists, which are built from a collection of physics constructors. Each physics constructor implements a specific subset of particles and physics processes. A set of EM physics constructors are available (Table 1 ) with the Geant 4 release 9.3. Each constructor includes a full list of particles and their EM interactions and can be added to any modular Physics List. The first constructor listed in Table 1 uses default parameters for all EM processes and models. The first four constructors include only processes and models from the Standard EM package. These constructors have different models and parameters for multiple Coulomb scattering (MCS) and different values of step limitation parameters for ionization ${ }^{9)}$ á (default 0.2 ) and ñ (default $1 \mathrm{~mm}$ ). Other constructors in Table 1 are built on top of the "Option3" standard physics constructor by addition of models from the Low-energy package for gamma and electrons below $1 \mathrm{GeV}$. More variants of Physics Lists are available with Geant 4 extended and advanced examples. ${ }^{25)}$

The main advantage of using these physics constructors is the fact that they are intensively validated, so these components of Physics Lists are more reliable and the accuracy of results are better predicted.

Table 1 List of EM physics constructors in Geant4 9.3

\begin{tabular}{ll}
\hline Constructor & Comments \\
\hline G4EmStandardPhysics & Default \\
minimamStandardPhysics_option1 & $\begin{array}{l}\text { á=0.8 for } \mathrm{e}^{ \pm} \\
\text {"ApplyCuts” option }{ }^{8)} \text { on, } \\
\text { G4WentzelVIModel of MCS } \\
\text { and single scattering for } \mu^{ \pm}\end{array}$ \\
G4EmStandardPhysics_option3 & $\begin{array}{l}\text { tight MCS step limitation; } \\
\text { increased number of bins in } \\
\text { all tables to 220; } \tilde{\mathrm{n}}=0.1 \mathrm{~mm}\end{array}$ \\
& $\begin{array}{l}\text { for } \mathrm{e}^{ \pm}, 0.05 \text { for } \mu^{ \pm} \text {and ha- } \\
\text { drons, } 0.02 \text { for ions; ICRU73 } \\
\text { based ion model }{ }^{22}\end{array}$ \\
& $\begin{array}{l}\text { Option3, Livermore models } \\
\text { for ã and } \mathrm{e}^{-}\end{array}$ \\
Option3, Livermore models \\
for ã and $\mathrm{e}^{-}$, polarization for ã \\
Option3, Penelope models for \\
G4EmLivermorePolarizedPhysics \\
ã and $\mathrm{e}^{ \pm}$ \\
Option3, DNA models for \\
low-energy interactions
\end{tabular}

\section{Helper Classes}

Several helper classes are available in Geant4 EM, which may be useful in various user applications:

- G4EmCalculator providing an interface to stopping powers, cross sections and ranges, allowing to compute these values without running a simulation; ${ }^{26}$

- G4EmProcessOptions providing an easy method to setup EM options from user Physics Lists;

- G4EmConfigurator providing an easy method to configure EM models per particle, energy range, and geometry region; 
- G4EmSaturation is a class to compute Birks correction to energy loss;

- G4ElectronIonPair is a class to sample ionization clusters in gaseous or semiconductor detectors.

The usage of these classes is not mandatory. However, user code may be significantly simplified if these classes are employed. Geant4 extended and advanced examples demonstrate how helper classes can be applied.

\section{Infrastructure Upgrade}

Recently, the software for handling internal tables for stopping powers, ranges, and cross sections has been improved significantly. ${ }^{8)}$ The main goal was to improve the precision of interpolation of data points without increasing the memory used by the Geant 4 application. This was achieved by the introduction of cubic spline. ${ }^{10)}$ In the EM design, the handling of tables is performed in generic classes described above. The benefits of the upgrade are available for all EM sub-packages and some hadronic sub-packages, which use the same methods to handle data.

An initialisation of a simple Geant 4 application is very fast but for a complex geometry the size of the prepared tables is large and their filling may take a considerable amount of time. It was identified that the most significant part of the CPU time at initialisation was spent for conversion ${ }^{1)}$ of Geant 4 cuts in range to production thresholds in energy. The optimisation of the software for Geant4 9.3 reduces initialisation time significantly (Table 2).

Table 2 CPU time for initialisation of Geant4 EM physics (default constructor) for a setup with 289 materials

\begin{tabular}{ccc}
\hline Geant4 version & AMD 32-bit & AMD 64-bit \\
\hline 9.2 & $147 \mathrm{~s}$ & $179 \mathrm{~s}$ \\
9.3 & $51 \mathrm{~s}$ & $56 \mathrm{~s}$ \\
\hline
\end{tabular}

\section{Recent EM Developments}

In this chapter selected developments are described, which are already available with Geant4 version 9.3 or are planned for the coming version 9.4 (December 2010).

\section{Relativistic Models}

A common approach for the relativistic $(\mathrm{E}>1 \mathrm{GeV})$ models of bremsstrahlung and gamma conversion to $\mathrm{e}^{+} \mathrm{e}^{-}$ pairs for electrons, muons, and hadrons has been developed. $^{5,6,8)}$ Initial differential cross sections are integrated in a similar way at initialisation taking into account production thresholds (cuts). The resulting restricted cross sections and energy losses are used at run time. ${ }^{9)}$ For electron models ${ }^{27}$ the classical approach ${ }^{28)}$ was implemented including the density effect suppression. The updated computation of the Landau-Pomeranchuk-Migdal (LPM) effect fits the data ${ }^{29)}$ much better than the previous Geant4 model (Fig. 1). These new models are included in all physics constructors described in Table 1 and are used in Monte Carlo simulation productions for LHC.

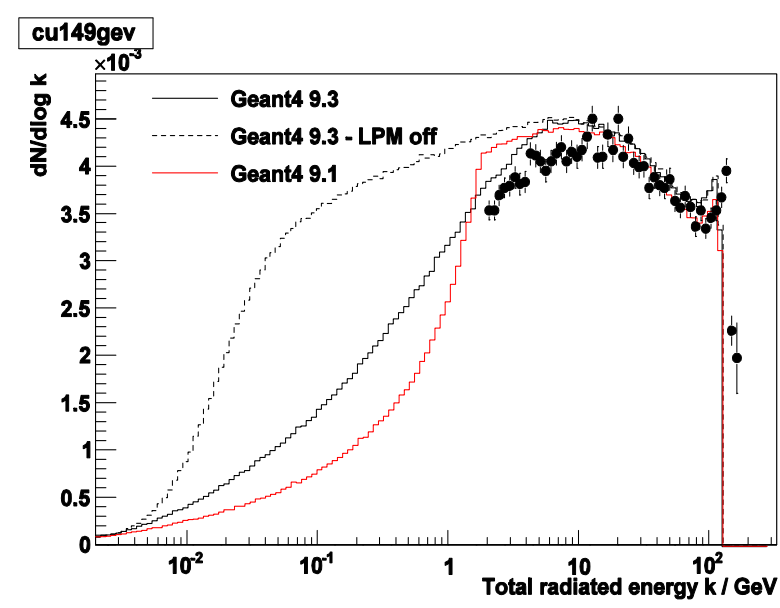

Fig. 1 Bremsstrahlung spectrum for $149 \mathrm{GeV}$ electron beam on a Copper target $\left(4 \% \mathrm{X}_{0}\right)$ : points - CERN data, ${ }^{29)}$ histograms Geant4 simulation results, 9.3 is in agreement with the data.

\section{Multiple and Single Scattering Models}

The MCS process is an important component of any Monte Carlo including Geant4. ${ }^{12)}$ At high energy it is limiting the spatial resolution of detectors. Scattering of electrons defines energy transport via geometrical boundaries directly affecting various detector responses. The default Geant4 MCS model ${ }^{30)}$ has been upgraded ${ }^{31)}$ for Geant4 version 8.0 (December 2006) due to a problem in electron scattering. ${ }^{32)}$ Later alternative models of single and multiple scattering have been added. ${ }^{11,12,33)}$ The main goal of these additions was to provide a choice between speed and precision specifically for different particles. Higher precision is achieved by simulation with smaller steps, which leads to an increase of the CPU time. Incorporation of corrections like spin term or nuclear form factor should be done depending on a particle type, also MCS parameters can also be optimized per particle. Thus, since Geant4 9.3 MCS processes used in EM constructors (Table 1) for electrons, muons, and hadrons are different, for each process the corresponding set of models and its parameters are defined.

To reach maximal precision the new Goudsmit- Saunderson MCS model for electrons and positrons has been developed, ${ }^{11)}$ which is fully based on theory and reusing experience and prescriptions of PENELOPE ${ }^{34)}$ and EGSnrc. ${ }^{35)}$ This model provides the best results for the Fano cavity test (Fig. 2) and for other benchmarks but is significantly slower than the default model. Thus, for the newest Geant4 version 9.4beta this model is included only in Livermore and Penelope physics constructors.

\section{New Models of Ionisation}

Recently intensive developments have been carried out to improve existing models of ionisation and to design new models including ionisation of magnetic monopole ${ }^{10)}$ and ionisation of ions heavier than Helium ${ }^{18)}$ using ICRU 73 data. The last model mainly focuses on the precise simulation of the transport of ions used in radiation therapy. ${ }^{36)}$

As the standard model for ion ionisation, the new model uses the effective charge approach, ${ }^{37)}$ where the ion charge 
state is considered in average only. But in some applications, like microdosimetry and ion beam transport in vacuum, this approach is not applicable. A set of new models have been developed ( 9.4 beta), which uses a dynamic ion charge instead of an effective charge. In that case the charge state should be defined by a special process of the charge exchange between an ion and the media. The demonstration of these new models will be available with Geant4 "TestEm7" extended EM and “microbeam” advanced examples.

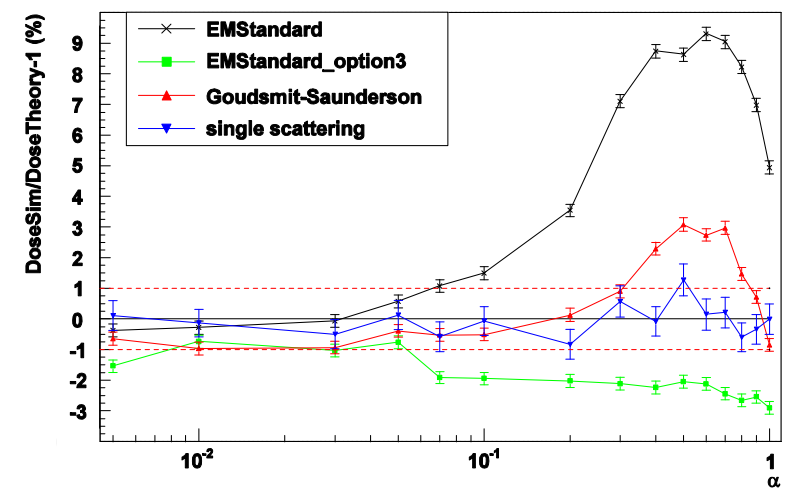

Fig. 2 Fano cavity test results ${ }^{31)}$ as a function of á (step limit parameter) for different MCS options for Geant4 9.4beta.

\section{DNA Physics Model}

Geant4 has been used in recent years for the simulation of experimental setups at the physics-biology frontier, including for example the design of high-resolution irradiation beam facilities at sub-micrometre size ${ }^{38}$ ) and dosimetry at the single-cell scale in realistic geometrical models of biological cells. ${ }^{39)}$ The Geant4 toolkit is currently being extended for microdosimetry simulation capabilities in biological media in the framework of the Geant4-DNA project, ${ }^{17,40)}$ originally initiated by the European Space Agency (ESA) for the modelling of biological effects of ionising radiation on astronauts at the DNA scale during manned long-duration space exploration missions. The Geant4-DNA extension is a component of the Low-energy EM package. It includes discrete EM physics models applicable to electrons, protons, neutral Hydrogen, neutral Helium and its charged states $\left(\mathrm{He}^{+}, \mathrm{He}^{2+}\right)$ in liquid water, the main component of biological media. Geant4-DNA processes simulate explicitly all interactions on a step-by-step basis in order to reconstruct precisely track structures of ionizing particles at nanometre scale, the size of DNA molecules.

Such computations require high computing performance and they are usually used at low energies (down to a few eV) and applied to small target volumes only, such as biological cells and sub-cellular volumes. In addition, Geant4 EM condensed-random-walk processes, ${ }^{1,9)}$ which cover complete energy range do not simulate explicitly all interactions with such precision and are much less demanding in computing performance. Consequently, these condensed-random-walk processes could be used to simulate the radiation environment surrounding astronauts in space, taking into account high energy incoming cosmic particles and modelling their interactions within spacecraft material, until they finally reach the target cells of interest, where Geant4-DNA discrete processes would take over.

The possibility to combine discrete and condensed processes in selected energy ranges and geometry regions is a new feature of the software design presented above. The combination of the Standard and Geant4-DNA EM models has been recently implemented in the Physics List of Geant4 "microdosimetry" advanced example, which illustrates how to perform in practice this combination of processes for a simple application case. This example proposes to simulate the irradiation of a thin liquid water slab, placed in the "world" mother volume, also filled with liquid water. Geant4-DNA processes only are activated within the slab.

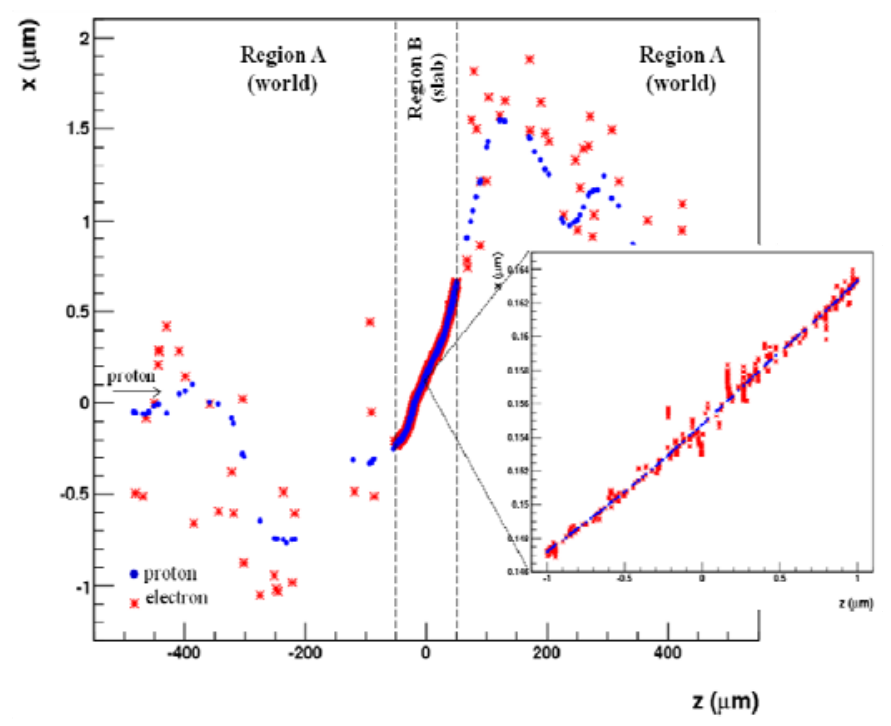

Fig. 3 Effect of combination of processes obtained with the "microdosimetry" advanced example. The 2D projected track of a 10 $\mathrm{MeV}$ proton in liquid water is shown. DNA processes are enabled inside a $100 \mu \mathrm{m}$ slab. Note the scale of the vertical axis is much smaller than the scale on the horizontal axis. The inset shows a magnification of secondary tracks inside the slab.

A trajectory of a single incident $10 \mathrm{MeV}$ proton on the slab is shown in Fig. 3, where two regions are used: the default G4Region (named “A”) and another G4Region (named "B") associated to this slab. Standard EM processes are activated in G4Region "A". On the contrary, proton and electron Geant4-DNA processes are activated in G4Region "B". They are ionisation and excitation for both protons and electrons and elastic scattering for electrons. Because these Low-energy processes have upper applicability limit, the Standard EM processes are also activated in "B" for high energy particles, using a lower energy applicability limit of $10 \mathrm{MeV}$ for protons and $1 \mathrm{MeV}$ for electrons, respectively.

The incident $10 \mathrm{MeV}$ proton is shot from G4Region "A" in the direction of the slab (Fig. 3). Inside the slab very detailed simulation of protons interactions and secondary particle transport is performed. Once the incident proton has left the water slab, it returns into G4Region "A" where only Standard EM processes are enabled. The possibility to combine such processes responds to a requirement of having 
Geant4 Physics processes able to work at different sizes and energy scales in a single simulation application.

\section{Recent Validation Results}

Validation of Geant4 EM software is a continuing task to the Geant4 EM working groups and software for validation and verification of EM packages has been developed. ${ }^{41)}$ Validations are performed for each modification of EM software and for each Geant4 version. Therefore software for automatic running and presenting of results has been designed. In parallel, number of specialized benchmarks was carried out recently.

The validation of the cross sections and stopping powers of Geant4 EM physics models was first done ${ }^{42)}$ versus NIST data. In this work it was concluded that Geant 4 photon cross sections agree with NIST within 3\% and electron stopping powers within $5 \%$. After the migration to common design a new validation of photon cross sections versus various databases was published ${ }^{26)}$ which demonstrated general good agreement with the data for both the Standard and Low-energy models. Also some problems were identified mainly when databases do not agree with each other.

The validation of the electron dose deposition in semi-infinite media in different materials from Be to $U$ has been published. ${ }^{43,44)}$ The primary beam energy was from $0.05 \mathrm{MeV}$ to $1 \mathrm{MeV}$ and primary beam angles from zero to 60 degrees. The absolute dose and longitudinal dose profile were measured. It was shown that results depend mainly on MCS model and are practically independent on ionization or bremsstrahlung. The Geant 4 accuracy is about 3\% for total energy deposition and is within $10 \%$ for the dose profile for majority of physics constructors. The worst precision is obtained with the Option 1 constructor where simplified step limitation is used.

Another benchmark comes from medical applications of electron/gamma transport for the simulation of ionization chamber. $^{35)}$ The Fano theorem predicts with the absolute accuracy the dose deposition in a cavity filled with vapor phase of surrounding condensed material. The current results of Geant4 benchmarking ${ }^{41)}$ are on level of 3\% for the default model and 1\% for the most precise one (Fig. 2).

The recent benchmark for electron scattering of $13 \mathrm{MeV}$ and $20 \mathrm{MeV}$ on various thin targets was published ${ }^{45)}$. The comparisons were performed ${ }^{46)}$ with predictions of different Monte Carlo systems EGSnrc, PENELOPE, and Geant4. Results were in statistical agreement with the data except Geant4 predictions for low-Z targets which provides up to $10 \%$ overestimates. After this analysis Urban MCS model was tuned for all electron data excluding the data from this benchmark, which gives now satisfactory results. The tuned version of the model is available with the Geant4 version 9.3. Also the most recent version (9.4 beta) of Goudsmit- Saunderson MCS model ${ }^{11)}$ provides good results.

Another dedicated benchmark ${ }^{47)}$ has been performed for EGSnrc, PENELOPE, and Geant4 using the most precise existing data for low-energy electron bremsstrahlung ${ }^{48,49)}$ for $10-30 \mathrm{MeV}$ electron beams in thick targets of $\mathrm{Be}, \mathrm{Al}$, and $\mathrm{Pb}$. Geant4 results are within 3 standard deviations at all energies and angles. It is shown that the Standard EM model overestimates the photon yield compared to the Livermore model. For the total photon yield the accuracy of the data is $3 \%$, EGSnrc results agree within 1 standard deviation, Penelope and Geant4 agree within 2 standard deviations. For the high energy the benchmarking data measured at SLAC ${ }^{50}$ and $\mathrm{CERN}^{29)}$ are available. Only new model ${ }^{8,27)}$ from the Standard EM package implements LPM and density suppressions correctly (Fig. 1).

Geant4-DNA Physics models for microdosimetry in liquid water were recently quantitatively compared $^{40)}$ to experimental data in water collected from the literature. In particular, the agreement between Geant4-DNA total and differential cross section models and the reference data has been evaluated using the Kolmogorov-Smirnov statistical test. For this evaluation, experimental data acquired in water vapor were used as direct measurements in the liquid phase are not available. Comparisons with several international recommendations (such as the recommendations of the ICRU reports) on collision stopping powers and on stopping cross sections have also been performed.

\section{Summary}

Since Geant4 version 9.3 both EM packages, Standard and Low-energy, follow a common interface for processes and models. It is possible to use in the same run ultra-relativistic, relativistic, and low-energy models simultaneously for optimal precision and speed. Validation procedures for EM physics are established. The main validation results are available via EM physics web. ${ }^{25)}$

\section{Acknowledgments}

This work was supported in part by ESA TRP contracts 21435/08/NL/AT, 22712/09/NL/AT, and 22839/10/NL/AT; RFBR grant 09-02-91065 and CNRS grant PICS-4865 and ANR contract ANR-09-BLAN-0135-01.

\section{References}

1) S. Agostinelli, et al., "Geant4 - a simulation toolkit," Nucl. Instr. Meth. Phys. Res., A506, 250 (2003).

2) J. Allison et al., "Geant4 developments and applications," IEEE Trans. Nucl. Sci., 53, 270 (2006).

3) K. Lassila-Perini, L.Urban, "Energy loss in thin layers in GEANT,” Nucl. Instr. Meth. Phys. Res., A362, 416 (1995).

4) J. Apostolakis, et al., "An implementation of ionisation energy loss in very thin absorbers for the Geant 4 simulation package," Nucl. Instr. Meth. Phys. Res., A453, 597 (1995).

5) H. Burkhardt, et al., "Geant4 standard electromagnetic package for HEP applications,” Nuclear Science Symposium Conference Record 2004, IEEE, Volume 3, pp.1907-1910.

6) A.G. Bogdanov, et al., alnovdanovlume 3, pp.190oduction and interaction of muons,nsIEEE Trans. Nucl. Sci,. 53, 513 (2006).

7) J. Apostolakis, et al., "The performance of the Geant4 standard EM package for LHC and other applications,” J. Phys.: Conf. Ser. 119, 032004 (2008).

8) V. N. Ivanchenko, et al., "Recent Progress of Geant4 Electromagnetic Physics and Readiness for the LHC Start," PoS (ACAT2008), 108, (2008).

9) J. Apostolakis, et al., "Geometry and physics of the Geant4 
toolkit for high and medium energy applications,” Rad. Phys. Chem., 78, 859 (2009).

10) A. Bagulya, et al., "Heavy-particle energy loss simulation using the Geant4 toolkit,” Bull. Lebedev Phys. Inst., 36, 29 (2009).

11) O. Kadri, et al., "Incorporation of the Goudsmit-Saunderson electron transport theory in the Geant 4 Monte Carlo code," Nucl. Instr. Meth. Phys. Res., B267, 3624 (2009).

12) V. N. Ivanchenko, et al., "Geant4 models for simulation of multiple scattering,” J. Phys: Conf. Ser., 219, 032045 (2010).

13) J. Apostolakis et al., Geant4 low energy electromagnetic models for electrons and photons, CERN-OPEN-99-034, INFN/AE-99/18 (1999).

14) S. Chauvie, et al., "Geant4 low energy electromagnetic physics,” Nuclear Science Symposium Conference Record 2004, IEEE, Volume 3, Oct 2004. pp. 1881-1885.

15) S. Guatelli, et al., "Geant4 Atomic Relaxation," IEEE Trans. Nucl. Sci., 54, 585 (2007).

16) H. B. Abdelouahed, et al., "New Geant4 Cross Section Models for PIXE Simulation,” Nucl. Instr. Meth. Phys. Res., B267, 37 (2008).

17) S. Incerti, et al., “The Geant4-DNA project,” Int. J. Model. Simul. Sci. Comput., 1[2], 157 (2010).

18) A. Lechner, V. N. Ivanchenko, J. Knobloch, "Validation of recent Geant4 physics models for application in carbon ion therapy,” Nucl. Instr. Meth. Phys. Res., B268, 2343 (2010).

19) D. Cullen, J. H. Hubbell, L. Kissel, The evaluated photon data library, Report UCRL-50400, Vol. 6 (1997).

20) S. T. Perkins, D. E. Cullen, S. M. Seltzer, Tables and graphs of electron-interaction cross-sections from $10 \mathrm{eV}$ to $100 \mathrm{GeV}$ derived from the LLNL evaluated electron data library (EEDL), $Z=1-100$, Report UCRL-50400, Vol. 31 (1997).

21) F. Salvat, et al., "PENELOPE-2008: A Code System for Monte Carlo Simulation of Electron and Photon Transport," Nuclear Energy Agency, ISBN 978-92-64-99066-1 (2009).

22) S. T. Perkins, et al., Tables and graphs of atomic sub-shell and relaxation data derived from the $L L N L$ evaluated atomic data library (EADL), $Z=1-100$, Report UCRL-50400, Vol. 30 (1997).

23) M. Maire, J. Jacquemier, "Electron ranges,” 11th Geant4 workshop, LIP, Lisbon, Portugal, 9-14 October 2006.

24) C. Zacharatou, et al., "Range validation for electrons, protons and alpha particles," 14th Geant4 workshop, INFN/LNS, Catania, Italy, 15-22 October 2009.

25) http://cern.ch/geant4/collaboration/working_groups/electroma gnetic/

26) G. A. P. Cirrone, et al., "Validation of the Geant4 electromagnetic photon cross-sections for elements and compounds," Nucl. Instr. Meth. Phys. Res., A618, 315-322 (2010).

27) A. Schaelicke, et al., "Improved description of bremsstrahlung for high-energy electrons in Geant4,” IEEE NSS conference, Dresden, Germany, 19-25 October 2008, NSS N37-1.

28) M. N. Ter-Mikaelian, High-energy electromagnetic processes in condensed media, Wiley (1972).

29) H. D. Hansen, et al., "Landau-Pomeranchuk-Migdal effect for multi-hundred GeV electrons,” Phys. Rev., D69, 032001 (2004).

30) L. Urban, A multiple scattering model in Geant4, Preprint CERN-OPEN-2006-077 (2006).
31) S. Elles, V. N. Ivanchenko, M. Maire, L. Urban, "Geant4 and Fano cavity test: where we are?” J. Phys.: Conf. Ser., 102, 012009 (2008).

32) E. Poon, F. Verhaegen, "Accuracy of the photon and electron physics in Geant4 for radiotherapy applications," Med. Phys. 32, 1696-1711 (2005).

33) M. H. Mendenhall, R. A. Weller, “An algorithm for computing screened Coulomb scattering in Geant4," Nucl. Instr. Meth. Phys. Res., B227, 420-430 (2005).

34) J. M. Fernandez-Varea, et al., "On the theory and simulation of multiple scattering of electrons," Nucl. Instr. Meth. Phys. Res., B73, 447-473 (1993).

35) I. Kawrakow, "Accurate condensed history Monte Carlo simulation of electron transport. II. Application to ion chamber response," Med. Phys., 27[3], 499 (2000).

36) T. Toshito, et al., "New Geant4 electromagnetic physics developments for ion therapy applications," Proc. Int. Conf. On Supercomputing in Nuclear Applications and Monte Carlo (SNA+MC 2010), Tokyo, Japan, Oct. 17-21, 2010 (2010).

37) J. F. Ziegler, J. M. Manoyan, "The stopping of ions in compounds,” Nucl. Instr. Meth. Phys. Res., B35, 215 (1988).

38) F. Andersson, et al., "A detailed ray-tracing simulation of the high resolution microbeam at the AIFIRA facility,” Nucl. Instr. Meth. Phys. Res., B266, 1653-1658 (2008).

39) S. Incerti, et al., "Monte Carlo dosimetry for targeted irradiation of individual cells using a microbeam facility," Rad. Prot. Dos. 133[1], 2-11 (2009).

40) S. Incerti, et al., "Comparison of GEANT4 very low energy cross section models with experimental data in water," Med. Phys., 37[9], 4692-4708 (2010).

41) J. Apostolakis, et al., "Validation and verification of Geant4 standard electromagnetic physics,” J. Phys: Conf. Ser., 219, 032044 (2010).

42) K. Amako, et al., "Comparison of Geant4 electromagnetic physics models against NIST reference data," IEEE Trans. Nucl. Sci., 52, 910-918 (2005).

43) O. Kadri, et al., "Geant4 simulation of electron energy deposition in extended media," Nucl. Instr. Meth. Phys. Res., B258, 381-387 (2007).

44) A. Lechner, M. G. Pia, "Validation of Geant4 low energy processes,” IEEE Trans. Nucl. Sci., 56, 398-416 (2009).

45) C. K. Ross, et al., "Measurement of multiple scattering of 13 and $20 \mathrm{MeV}$ electrons by thin foil,” Med. Phys., 35[9], 4121-4131 (2008).

46) B. A. Faddegon, et al., "The accuracy of EGSnrc, Geant4 and PENELOPE Monte Carlo systems for the simulation of electron scatter in external beam radiotherapy,” Phys. Med. Biol., 54, 6151-6153 (2009).

47) B. A. Faddegon, et al., "Benchmarking of Monte Carlo simulation of bremsstrahlung from thick targets at radiotherapy energies," Med. Phys., 35, 4308-4317 (2008).

48) B. A. Faddegon, et al., "Forward directed bremsstrahlung of 10-30 MeV electrons incident on thick targets of $\mathrm{Al}$ and $\mathrm{Pb}$," Med. Phys., 17, 773-785 (1990).

49) B. A. Faddegon, et al., "Angular distributions of bremsstrahlung of $15 \mathrm{MeV}$ electrons incident on thick targets of $\mathrm{Be}, \mathrm{Al}$ and Pb,” Med. Phys., 18, 727-739 (1991).

50) P. L. Antony, et al., "An accurate measurement of the LPM effect,” Phys. Rev. Lett., 75, 1949-1952 (1995). 